\title{
The Sasa quelpaertensis Leaf Extract Inhibits the Dextran Sulfate Sodium-induced Mouse Colitis Through Modulation of Antioxidant Enzyme Expression
}

\author{
Yiseul Yeom, Yuri Kim \\ Department of Nutritional Science and Food Management, College of Health Sciences, Ewha Womans University, Seoul, Korea
}

\begin{abstract}
Background: Oxidative stress plays an important role in the pathogenesis of inflammatory bowel disease. The objective of this study is to investigate the protective effect of Sasa quelpaertensis leaf extract (SQE) against oxidative stress in mice with dextran sulfate sodium (DSS)-induced colitis.

Methods: Mice were treated with SQE $(100 \mathrm{mg} / \mathrm{kg}$ or $300 \mathrm{mg} / \mathrm{kg}$ body weight) by gavage in advance two weeks before inflammation was induced. Then, the mice were administered with 2.5\% DSS in drinking water for 7 days and normal drinking water for 7 days between two DSS treatment. Disease activity index values, gut motility, and severity of the resulting oxidative DNA damage were analyzed. The antioxidant effect of SQE was evaluated by measuring malondialdehyde (MDA) and superoxide dismutase (SOD) activity in plasma samples. Catalase activity and expressions levels of glutathione peroxidase 1 (Gpx1), SOD1, and SOD2 were also detected in colon tissues. Results: Administration of SQE significantly reduced the severity of DSS-induced colitis compared to the control (Ctrl) group. Levels of 8-oxo-dG, an oxidative DNA damage marker, were significantly lower in the SQE group compared to the untreated DSS Ctrl group. In the SQE (300 mg/kg) group, MDA levels were significantly lower, while SOD and catalase activity levels in the plasma samples were significantly higher compared with the DSS Ctrl group. The expression levels of the antioxidant enzymes, SOD2 and Gpx1, were significantly higher, while the levels of SOD 1 expression were lower, in the colon tissues of the DSS Ctrl group compared with those of the Ctrl group. In contrast, administration of SQE significantly down-regulated SOD2 and Gpx1 expressions and up-regulated SOD1 expression. Conclusions: These results indicate that SQE efficiently suppresses oxidative stress in DSS-induced colitis in mice, and its action is associated with the regulation of antioxidant enzymes.
\end{abstract}

(J Cancer Prev 2015;20:136-146)

Key Words: Sasa quelpaertensis leaf, Dextran sulfate, Colitis, Antioxidant

\section{INTRODUCTION}

Inflammatory bowel diseases (IBD), as well as Crohn's disease and ulcerative colitis (UC), are intestinal inflammatory disorders that lead to mucosal disruption and ulceration characterized by abdominal pain and diarrhea. ${ }^{1}$ The incidence rates and prevalence of IBD are high in Western countries, although the rates are beginning to rise in developing countries as well. ${ }^{2}$ Currently, the etiologies of IBD remain unclear, although it is known that they are commonly caused by a combination of factors, including genetic predisposition and environmental factors. ${ }^{1}$ Among the various causes of IBD, oxidative stress due to reactive oxygen species (ROS) is one of the most important factors in the genesis and progression of IBD. ${ }^{3}$ ROS also plays an important role in the malignant progression of tumor cells and carcinogenesis. ${ }^{4}$ During the progression of IBD, activated inflammatory cells produce large amounts of ROS. ${ }^{5}$ Several studies have reported that epithelial cell injury leading to the production of

Received June 13, 2015, Revised June 20, 2015, Accepted June 20, 2015

Correspondence to: Yuri Kim

Department of Nutritional Science and Food Management, College of Health Sciences, Ewha Womans University, 52 Ewhayeodae-gil, Seodaemun-gu, Seoul 120-750, Korea

Tel: +82-2-3277-4485, Fax: +82-2-3277-2862, E-mail: yuri.kim@ewha.ac.kr, ORCID: Yuri Kim, http://orcid.org/0000-0001-7606-8501

Copyright (C) 2015 Korean Society of Cancer Prevention

(c) This is an Open Access article distributed under the terms of the Creative Commons Attribution Non-Commercial License (http://creativecommons.org/licenses/by-nc/4.0) which permits unrestricted non-commercial use, distribution, and reproduction in any medium, provided the original work is properly cited. 
ROS has been detected in IBD patients and in an animal model of dextran sulfate sodium (DSS)-induced inflammation. ${ }^{6-8}$ The initial oxidative stress activates a defense mechanism that leads to detoxification of the ROS. However, the accumulation of oxidative stress can induce membrane damage and DNA adducts, and eventually can cause the degeneration of tissues and cellular transformation.?

Inflammatory injury to tissues can also induce excessive production of ROS. To counteract the harmful effects of ROS, the intestinal mucosa possesses an efficient antioxidant system. In this system, superoxide dismutase (SOD), catalase, and glutathione peroxidase (Gpx) play important roles. SOD converts the highly reactive superoxide anion, $\mathrm{O}_{2}{ }^{-}$, to the less reactive species, $\mathrm{H}_{2} \mathrm{O}_{2}$ and $\mathrm{O}_{2} \cdot{ }^{10} \mathrm{Gpx}$ and catalase normally remove $\mathrm{H}_{2} \mathrm{O}_{2}$ produced by SOD enzyme. ${ }^{11}$ In the intestinal mucosa of IBD patients, the balance of antioxidant enzyme levels has been found to be seriously impaired. ${ }^{12,13}$

To study IBD, a DSS-induced colitis model is generally used ${ }^{14}$ since it reflects many characteristics of IBD, including elevated levels of ROS and their metabolites. ${ }^{15}$ It is hypothesized that the study of ROS events may facilitate our understanding of the primary and downstream secondary pathophysiological mechanisms that mediate intestinal inflammation. ${ }^{16}$

Several antiinflammatory drugs including sulfasalazine (SSZ) are commonly colon specific drugs used for treatment IBD. ${ }^{17}$ However, these drugs had limitations and showed associated side effects. ${ }^{18,19}$ Therefore, recently plant extracts or blend tea from plant leaves for IBD management have been growing interest by their therapeutic effects. ${ }^{20-22}$ Natural herbal medicines have shown to be beneficial for the treatment of IBD and they also help to maintain healthy intestinal functions. ${ }^{23}$ In particular, a bamboo grass that is widely grown in Korea, China, and Russia, named Sasa leaves, has exhibited antiinflammatory, antidiabetic, and antipyretic properties. ${ }^{24}$ Among the known types of Sasa leaves, Sasa quelpaertensis Nakai is endemic to the area surrounding Mt. Halla on Jeju Island, Korea has exhibited antiinflammatory, antidiabetic, and antipyretic properties. Recent studies have reported that $S$. quelpaertensis Nakai have antidiabetic, antiobesity, antioxidative, and anticancer effects. ${ }^{25-27}$ S. quelpaertensis leaf extract (SQE) contains amino acids and polyphenols, including p-coumaric acid and tricin. p-coumaric acid has been shown to exert an antiinflammatory effect, while tricin has suppressed inflammatory-related carcinogenesis in experimental animals. $^{28,29}$

Considering the results of these previous studies, we hypothesize that SQE exerts an antioxidant effect in a mouse model of
DSS-induced colitis by protecting intestinal tissues and cells from oxidative stress. SQE may also help restore the balance among relevant antioxidant enzymes.

\section{MATERIALS AND METHODS}

\section{Extraction of Sasa quelpaertensis Nakai leaves}

S. quelpaertensis Nakai leaves were collected from Mt. Halla on Jeju Island in South Korea. Extractions were performed as previously described. ${ }^{30}$ Briefly, leaves $(1 \mathrm{~kg}$ ) were dried and washed in running water, were cleaned twice with deionized water, and then were dried and extracted with $70 \%$ ethanol for 48 hours at room temperature (RT). The SQE extract was subsequently filtered and dried on a rotary evaporator under reduced pressure. Following lyophilization, the extraction was crushed into a powder and was stored at $-20^{\circ} \mathrm{C}$ until needed.

\section{Animal groups and induction of dextran sulfate sodium-induced colitis}

Five-week-old male C57BL/6 mice (Central Lab. Animal Inc., Seoul, Korea) were maintained under standard laboratory conditions: $22^{\circ} \mathrm{C} \pm 2^{\circ} \mathrm{C}, 50 \% \pm 5 \%$ humidity, and 12 hours $/ 12$ hours light/dark cycles. Animals received a modified American Institute of Nutrition (AIN)-93G pellet diet (Unifaith Inc., Seoul, Korea). All mice were allowed to adapt to their new husbandry conditions for 1 week. The mice were randomized into five groups with 10 animals per group.

The five experimental groups included: i) control (Ctrl), ii) DSS-induced colitis (DSS Ctrl), iii) DSS $+100 \mathrm{mg} / \mathrm{kg}$ body weight (bw)/d SSZ (SSZ), iv) DSS + $100 \mathrm{mg} / \mathrm{kg} \mathrm{bw/d} \mathrm{SQE} \mathrm{(SQE} \mathrm{100),} \mathrm{v)} \mathrm{DSS}$ $+300 \mathrm{mg} / \mathrm{kg}$ bw/d SQE (SQE 300). The Ctrl group received a standard diet and normal drinking water. To induce colitis, mice were administered with 2.5\% DSS (molecular weight: 36-50 KD; MP Biomedicals, Costa Mesa, CA, USA) in drinking water for 7 days, followed by 7 days of untreated drinking water, and another 7 days of $2.5 \%$ DSS drinking water. The SSZ group was orally administered SSZ dissolved in distilled water for 21 days. Both SQE groups were pretreated with SQE for 14 days prior to DSS treatment. During the experimental period, bw and diet intake were recorded twice a week. After five weeks, all of the mice were sacrificed and plasma and tissue samples were collected and stored at $-80^{\circ} \mathrm{C}$. Animal care and experimental protocols for this study were approved by the Animal Care and Use Committee of Ewha Womans University (IACUC approval no: IACUC 14-070). 


\section{Disease activity index}

Disease activity index (DAI) score were calculated by adding combined scores for weight loss, stool consistency, and fecal bleeding, and dividing this sum by three. Mean values were assigned for stool consistency according to the presence of loose feces and watery diarrhea. For fecal bleeding was scored as normal, slightly bloody, and blood in whole colon compared to the Ctrl group. ${ }^{30}$ DAI scoring was performed from the start of DSS administration until the end of the experimental period.

\section{Histopathologic analysis and immunohistochemical detection of 8-oxo-dG}

For each collected colon tissue, fecal residue was removed, the tissue was gently rinsed in saline solution, and the tissue was fixed in 10\% neutral-buffered formaldehyde. After 24 hours, each tissue was embedded in paraffin. Cut sections $(4 \mu \mathrm{m})$ were stained with H\&E according to standard procedures. ${ }^{31}$

For immunohistochemical staining of 8-oxo-dG, sections were deparaffinized, rehydrated, and incubated overnight at $4^{\circ} \mathrm{C}$ with a primary anti-8 hydroxyguanosine antibody (Abcam, Cambridge, UK). After incubating the section with an appropriate secondary antibody at RT for 1 hour, the sections were washed, stained with 3,-3'-diaminobenzidine, and counterstained with hematoxylin for 1 minute. Slides were mounted using Permount mounting medium (Fisher Scientific, Pittsburgh, PA, USA). The percentage of positively stained cells among the total number of cells was evaluated in three randomly chosen fields and the mean value was recorded. ${ }^{30}$

\section{Measurement of gut transit time and gut motility}

To study gut motility, whole gut transit time was measured. ${ }^{32}$ Briefly, $300 \mu \mathrm{L}$ of a carmine red solution ( $3 \mathrm{~g}$ carmine in $50 \mathrm{~mL}$ of 0.5\% methylcellulose; Sigma Aldrich, St. Louis, MO, USA) was administered to each mouse by gavage. These mice were then placed in individual cages contained a white sheet on the bottom of the cage in order to distinguish the red stools from the normal stools. Total whole gut transit time was recorded following the first appearance of a red stool pellet. Gut motility was subsequently calculated as gut transit time divided by colon length.

\section{Measurement of plasma malondialdehyde, plasma superoxide dismutase activity, and colonic catalase activity}

To detect malondialdehyde (MDA), lipid peroxidation in plasma was measured with a TBARS Assay Kit (ZeptoMetrix,
Buffalo, NY, USA), according to the manufacturer's instructions. Plasma SOD activity was measured with a SOD Assay Kit (Cayman Chemical Company, Ann Arbor, MI, USA) according to the manufacturer's protocol.

Colon rectum tissues were collected and homogenized in 500 $\mu \mathrm{L}$ cold reaction buffer supplemented with a protease inhibitor cocktail (Sigma Aldrich). After centrifugation $\left(4^{\circ} \mathrm{C}, 15\right.$ minutes, $16,000 \times g$ ), supernatants were stored at $-80^{\circ} \mathrm{C}$. Catalase activity was detected by using an Amplex Red ${ }^{\mathbb{R}}$ Catalase Assay Kit (Invitrogen, Carlsbad, CA, USA.) according to the manufacturer's instructions.

\section{Western blot analysis}

Frozen whole colon tissues were homogenized in ice-cold PRO-PREP protein extraction solution (Intron Biotechnology, Seoul, Korea). Homogenates were centrifuged $\left(4^{\circ} \mathrm{C}, 12,000 \times g, 15\right.$ minutes) and supernatants were stored at $-80^{\circ} \mathrm{C}$. Heat-denatured tissue homogenates were separated using gradient (12\%-15\%) sodium dodecyl sulfate polyacrylamide gel electrophoresis. For western blot analysis, proteins were transferred to polyvinylidene difluoride membranes and were blocked with $5 \%$ skim milk or $3 \%$ bovine serum albumin $+2 \%$ skim milk in tris-buffered saline containing Tween-20 (TBST). Immunoblot analysis was performed using the following primary antibodies: SOD1 (Santa Cruz Biotechnology, Santa Cruz, CA, USA), SOD2 (Santa Cruz Biotechnology), Gpx1 (Abfrontier, Seoul, Korea), and $\alpha$-tubulin (Sigma Aldrich). After an overnight incubation at $4^{\circ} \mathrm{C}$, the membranes were washed three times with TBST and then were incubated with the appropriate secondary rabbit or mouse immunoglobulin G-conjugated horseradish peroxidase antibodies (Santa Cruz Biotechnology) at RT for 1 hour. After the membranes were washed three times, bound antibodies were detected using an enhanced chemiluminescence reagent (Animal Genetics Inc., Suwon, Korea).

\section{Statistical analysis}

Data shown are the mean \pm SEM for each group. Statistical analyses were performed using GraphPad PRISM software (GraphPad Software, San Diego, CA, USA). For multiple comparisons, one-way ANOVA with Tukey's post-hoc test was used. A $P$-value less than 0.05 was considered statistically significant. 


\section{RESULTS}

\section{Administration of Sasa quelpaertensis leaf extract suppresses the development of dextran sulfate sodium-induced colitis in mice}

DAI scores were higher in the DSS-induced group at day 19 compared to the Ctrl group (Fig. 1A). In addition, severe rectal bleeding, loose fecal, and weight loss during the second cycle of DSS were characterized in the DSS Ctrl group. In contrast, SSZ, SQE100, and SQE300 treatments were found to significantly suppress DAI scores by 33.3\% ( $P<0.05), 57.8 \%(P<0.001)$, and $72.2 \%(P<0.001)$ at day 19 compared to the Ctrl group. In particular, the mean DAI score of the SQE 300 group nearly achieved the mean DAI score of the Ctrl group. Furthermore, colorectal tissues from the Ctrl group exhibited normal histology, whereas colorectal tissues from the DSS Ctrl group exhibited severe inflammation, crypt distortion, and architectural abnormalities (Fig. 1B). However, in the SQE groups, crypt distortion was suppressed and only mild tissue damage was observed.

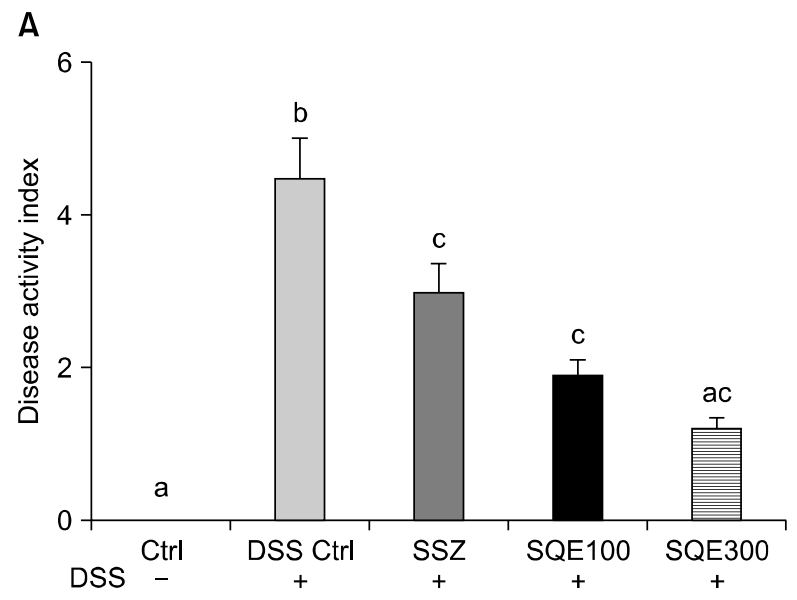

B
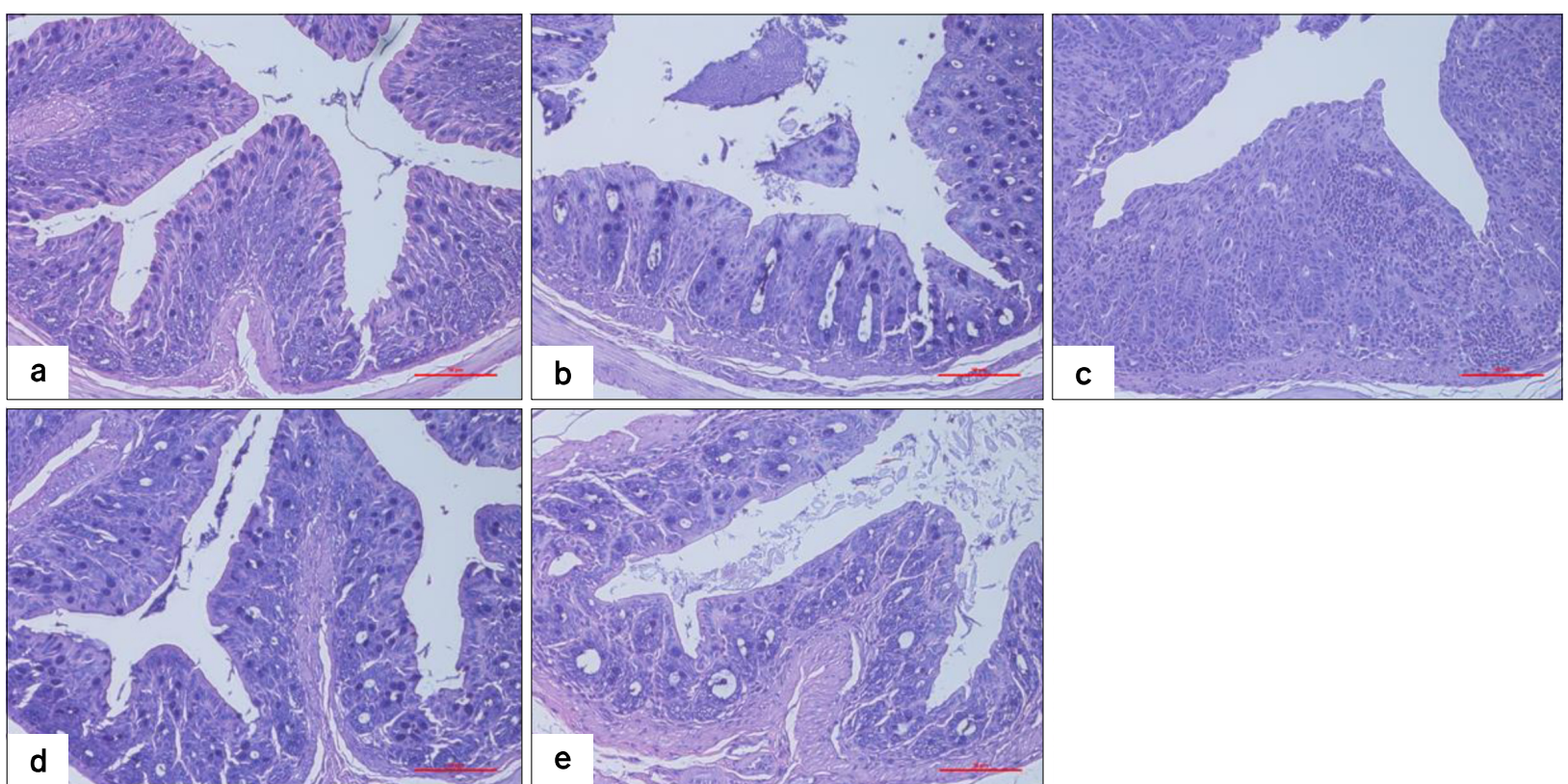

Figure 1. Administration of SQE suppresses the development of DSS-induced colitis in mice. (A) Disease activity index values were evaluated based on scores for body weight loss, stool consistency, and fecal bleeding for each of the groups. (B) Representative histologic damage in colorectal sections obtained from Ctrl (a), DSS Ctrl (b), SSZ (c), SQE 100 (d), and SQE 300 (e) mice. Colorectal tissues sections were stained with H\&E (scale bar: $50 \mu \mathrm{m}$ ). Data are shown to as the mean \pm SEM and were analyzed using one-way ANOVA and Tukey's post-hoc test $(P<0.05) ; \mathrm{n}=10$ mice per group. Different letters are used to indicate significant differences. SQE, Sasa quelpaertensis extract; Ctrl, control; DSS, dextran sulfate sodium; SSZ, sulfasalazine. 


\section{Sasa quelpaertensis leaf extract administration} apparently restored the gut motility in the dextran sulfate sodium-induced colitis mouse model

To determine the effects of SQE on gut motility, whole gut transit time was measured according to the first appearance of a carmine red stained stool (Fig. 2A). There was no significant difference in gut transit time between the groups although the administration of SQE groups tended to have a shorter time than the Ctrl group. When gut motility was determined by dividing the gut transit time by colon length, gut motility was found to be significantly reduced in the DSS Ctrl group compared to the Ctrl group (Fig. 2B), while gut motility for the SQE groups tended to be increased compared to the DSS Ctrl group. However, the differences were not statistically significant.

3. Sasa quelpaertensis leaf extract administration alleviates oxidative DNA damage caused by dextran sulfate sodium in the mouse colon

The reaction of an $\mathrm{OH}$ radical with a DNA guanosine base generates 8-oxo-dG. . $^{33}$ Thus, detection of 8-oxo-dG has been used as a marker of DNA damage. The percentage of positively stained epithelial cells with 8-oxo-dG was significantly higher in the colon tissues of the DSS Ctrl group (49.8\%) compared with the tissues from the Ctrl group (Fig. 3). SSZ treatment did not affect the number of 8-oxo-dG-positive cells compared to the DSS Ctrl group, while administration of SQE significantly reduced the percentage of 8-oxo-dG- positive cells compared to the DSS Ctrl

A

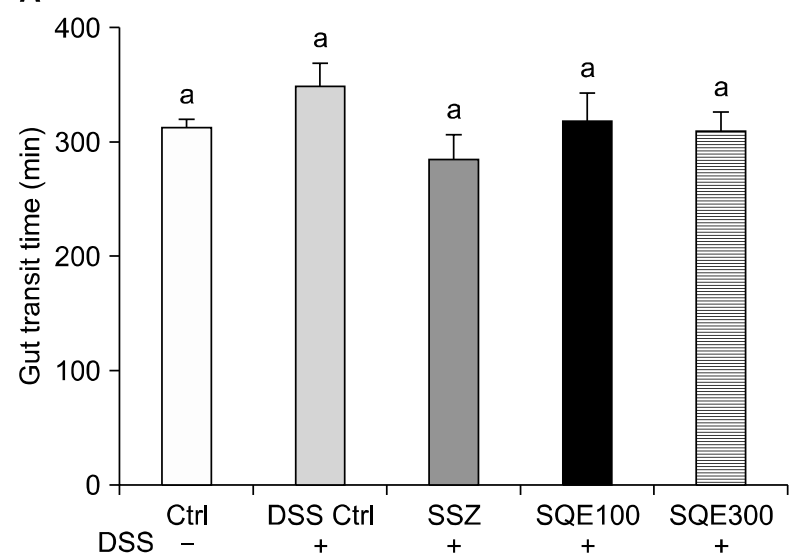

group $(P<0.05)$. In particular, the percentage of 8-oxo-dG-positive cells in the SQE 300 tissues was significantly decreased by $36.1 \%$ compared to that of the DSS Ctrl group tissues $(P<0.05)$, and this percentage was similar to that of the Ctrl group. Taken together, these results suggest that SQE treatment protects against DNA damage from DSS-induced colitis in mice.

\section{Sasa quelpaertensis leaf extract administration} regulates antioxidant enzyme activity in dextran sulfate sodium-induced colitis in mice

To evaluate the antioxidative capacity of SQE administration, MDA and SOD activity levels in plasma samples, and catalase activity in colon tissues were analyzed. MDA activity in the SQE 300 group was significantly lower by $28.1 \%$ compared with that of the DSS Ctrl group (Fig. 4A; $P<0.05$ ), and there it was no statistically significant difference between the MDA activity of the SSZ group and the DSS Ctrl group. Expression levels of SOD were lower by 20.7\% in the DSS Ctrl group compared with the Ctrl group (Fig. 4B), whereas the SOD levels were increased by $28.1 \%$ in the SQE 300 group compared to the DSS Ctrl group $(P<0.01)$. Both of these differences were significant. In combination, these results indicate that SQE protects the levels of SOD activity in the colon tissues of mice with DSS-induced colitis. Regarding the levels of catalase, the lowest levels were detected in the colon tissues of the DSS Ctrl group (Fig. 4C). In contrast, the catalase activity level of the SQE 300 group was significantly increased by 44.7\% compared with the DSS Ctrl group $(P<0.05)$. Overall, these results indicate that SQE regulates antioxidant enzyme activity against oxidative stress in DSS-induced colitis in mice.
B

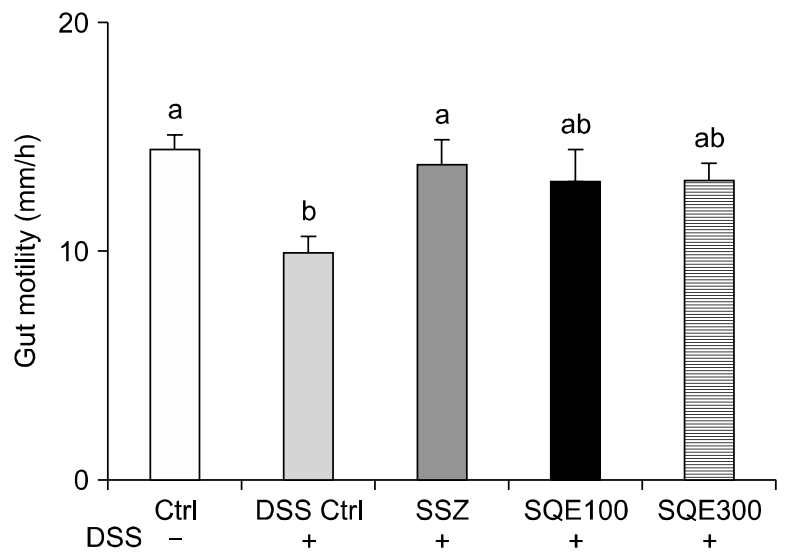

Figure 2. Administration of SQE alters gut motility in DSS-induced colitis model. (A) Gut transit time for all groups was analyzed by measuring the time of first development of red stools after carmine red administration. (B) Gut motility was estimated by the time and colon length for each group. Data are shown to as the mean \pm SEM and were analyzed using one-way ANOVA and Tukey's post-hoc test $(P$ $<$ 0.05); $\mathrm{n}=10$ mice per group. Different letters are used to indicate significant differences. SQE, Sasa quelpaertensis extract; DSS, dextran sulfate sodium; Ctrl, control; SSZ, sulfasalazine. 
A
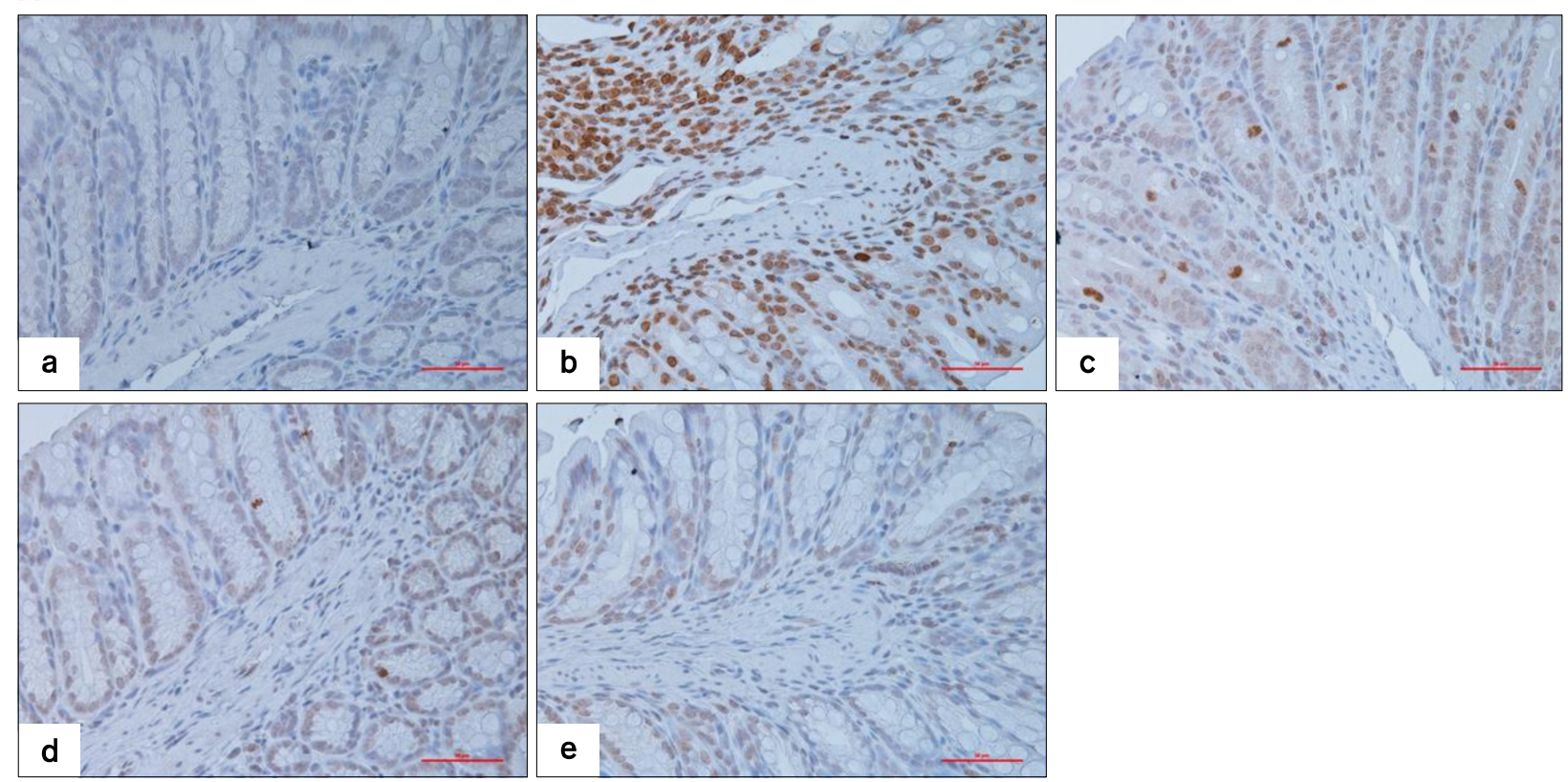

B

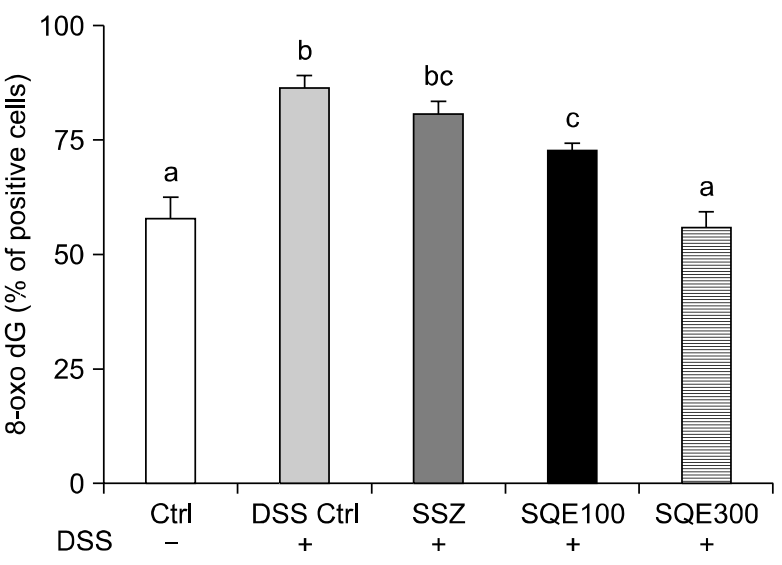

Figure 3. Administration of SQE alleviates oxidative DNA damage caused by DSS in the mouse colon. (A) Production of 8-oxo-dG was detected using immunohistochemistry in sections of colorectal tissue obtained DSS-induced colitis mice for: (a) Ctrl, (b) DSS Ctrl, (c) SSZ, (d) SQE 100, and (e) SQE 300 (a-e: $\times$ 400). (B) The percent of positive cells from 3 fields were counted and averaged. Scale bar: $50 \mu \mathrm{m}$. Data are shown to as the mean \pm SEM and were analyzed using one-way ANOVA and Tukey's post-hoc test $(P<0.05) ; \mathrm{n}=$ 10 mice per group. Different letters are used to indicate significant differences. SQE, Sasa quelpaertensis extract; Ctrl, control; DSS, dextran sulfate sodium; SSZ, sulfasalazine.

\section{Sasa quelpaertensis leaf extract administration regulates expression of superoxide dismutase and glutathione peroxidase 1 in the colon of mice treated with dextran sulfate sodium}

To investigate the role of SQE administration in regulating oxidative stress in a mouse model of DSS-induced colitis, the expression levels of several antioxidant enzymes, SOD1 (Cu-Zn SOD), SOD2 (Mn SOD), and Gpx1, were analyzed. For the DSS group, the levels of SOD1 expression decreased by $27.2 \%$ compared with the Ctrl group $(P<0.01)$. However, the SOD1 expression levels were significantly higher in both of the SQE groups compared to the DSS Ctrl group (Fig. 5A). SSZ treatment did not affect SOD expression. In contrast, the enzyme activity levels of SOD2 and Gpx1 in colon tissues from the DSS Ctrl group

were higher than those of the Ctrl group. In the SQE 300 tissues, the enzyme expressions of SOD2 and Gpx1 were significantly lower by $31.9 \%(P<0.001)$ and $27.3 \%(P<0.01)$, respectively, compared with the DSS Ctrl group (Fig. $5 \mathrm{~B}$ and $5 \mathrm{C}$ ). These results suggest that SQE administration regulates the expression levels of certain antioxidant enzymes that are involved in a DSS-induced colitis in mice.

\section{DISCUSSION}

In our previous studies, SQE treatment was shown to regulate levels of proinflammatory markers, mitogen-activated protein kinase signaling, and activation of NF-KB in a mouse model of DSS-induced colitis. ${ }^{30}$ In the present study, the antioxidative effect of SQE was also demonstrated in a mouse model of 
A

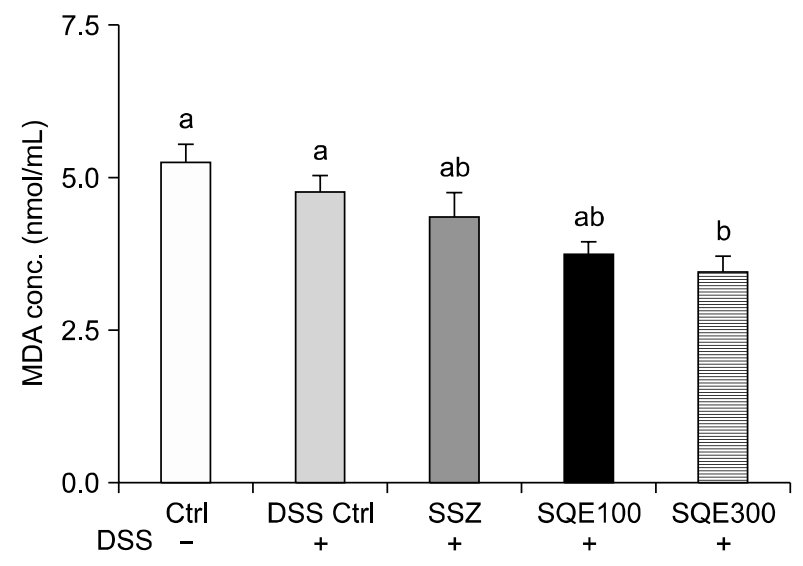

C

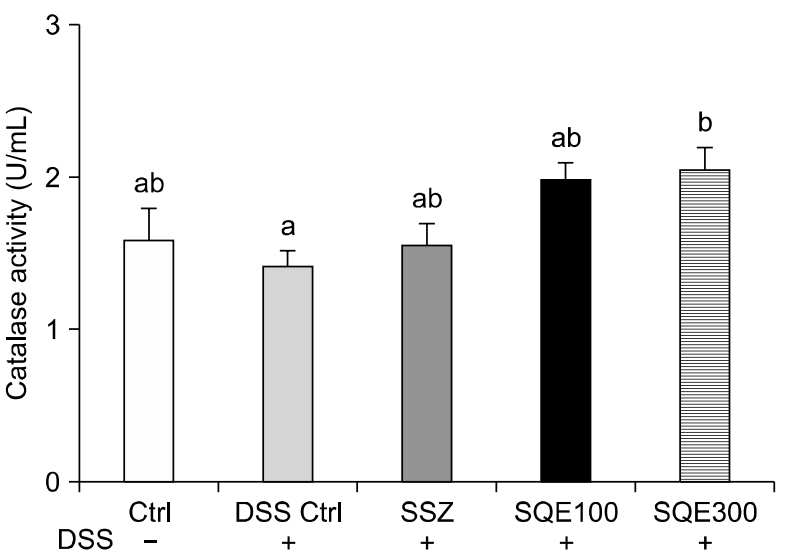

DSS-induced colitis. DSS administration effectively induces clinical features in mice that are similar to those of patients with IBD, including compromise of the integrity of the mucosal barrier that leads to intestinal epithelial cell inflammation and oxidative stress as a result of ROS generation. ${ }^{34} \mathrm{ROS}$ can either positively or negatively modulate an inflammatory response, and it plays an important role in the pathophysiology of IBD. ${ }^{35}$ Oxidative stress is considered to be critical in the tissue destruction in IBD and it affects carcinogenesis. ${ }^{36}$ Many studies have reported that animals subjected to DSS-induced colitis show increased level of oxidized protein in their plasma, as well as increased oxidative injury due to the inflammatory response. ${ }^{37.38}$ In the present study, SSZ was used as a drug control. SSZ has previously been characterized as a therapeutic agent for IBD, albeit with side effects that include ulcerogenic potential and additional oxidative stress. ${ }^{19.39}$ The dose of SSZ that was used in the present study represents a commonly used dose in animal studies. In the present study, SQE exhibited better protection from DNA damage and oxidative stress compared with SSZ, which indicated the therapeutic potential of SQE for inflammatory IBD.
B

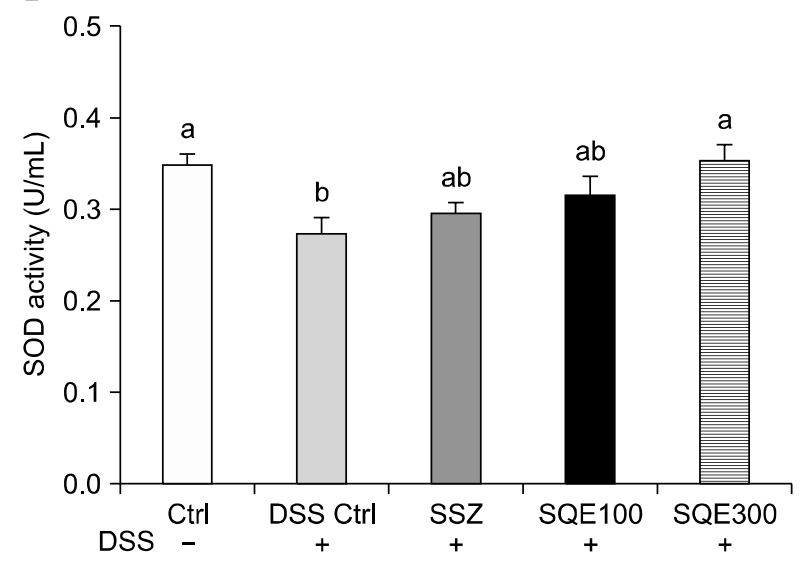

Figure 4. Administration of SQE regulates antioxidant enzyme activity in DSS-induced colitis mice. Levels of MDA (A) and SOD (B) were examined by commercial kit in plasma for all groups. (C) Catalase activity was examined in colon tissues by using a commercial kit. Data are shown to as the mean \pm SEM and were analyzed using one-way ANOVA and Tukey's post-hoc test $(P<0.05) ; \mathrm{n}=$ 10 mice per group. Different letters are used to indicate significant differences. SQE, Sasa quelpaertensis extract; DSS, dextran sulfate sodium; MDA, malondialdehyde; SOD, superoxide dismutase; conc., concentration; Ctrl, control; SSZ, sulfasalazine.

The observed increase in DAI values for the DSS Ctrl group is similar to that commonly observed for IBD patients. ${ }^{40}$ In addition, massive crypt distortion and architectural abnormalities that characterized the colon tissues of the DSS Ctrl group are consistent with those of a previous study, which indicated a severe inflammation response had developed ${ }^{30}$ However, in the present study, SQE treatment reduced the severity of colitis as evidenced by the suppressed DAI scores in the SQE group.

Gut transit time is a parameter that can be used to evaluate the functions of a gastrointestinal system. In the present study, gut transit time was evaluated in relation to inflammation. In the SQE groups, gut transit time was reduced, thereby implying that administration of SQE improves gut motility. However, since gut transit time can be affected by various factors, including nutrient absorption, physical activity, digestive ability, and eating time, additional studies are needed to confirm the present results in relation to these factors.

Increased levels of 8-oxo-dG have been detected in patients with IBD, and may be due to increased oxidation of DNA. ${ }^{41}$ Sheridan et al. ${ }^{33}$ also reported that high levels of 8-oxo-dG-positive 
A
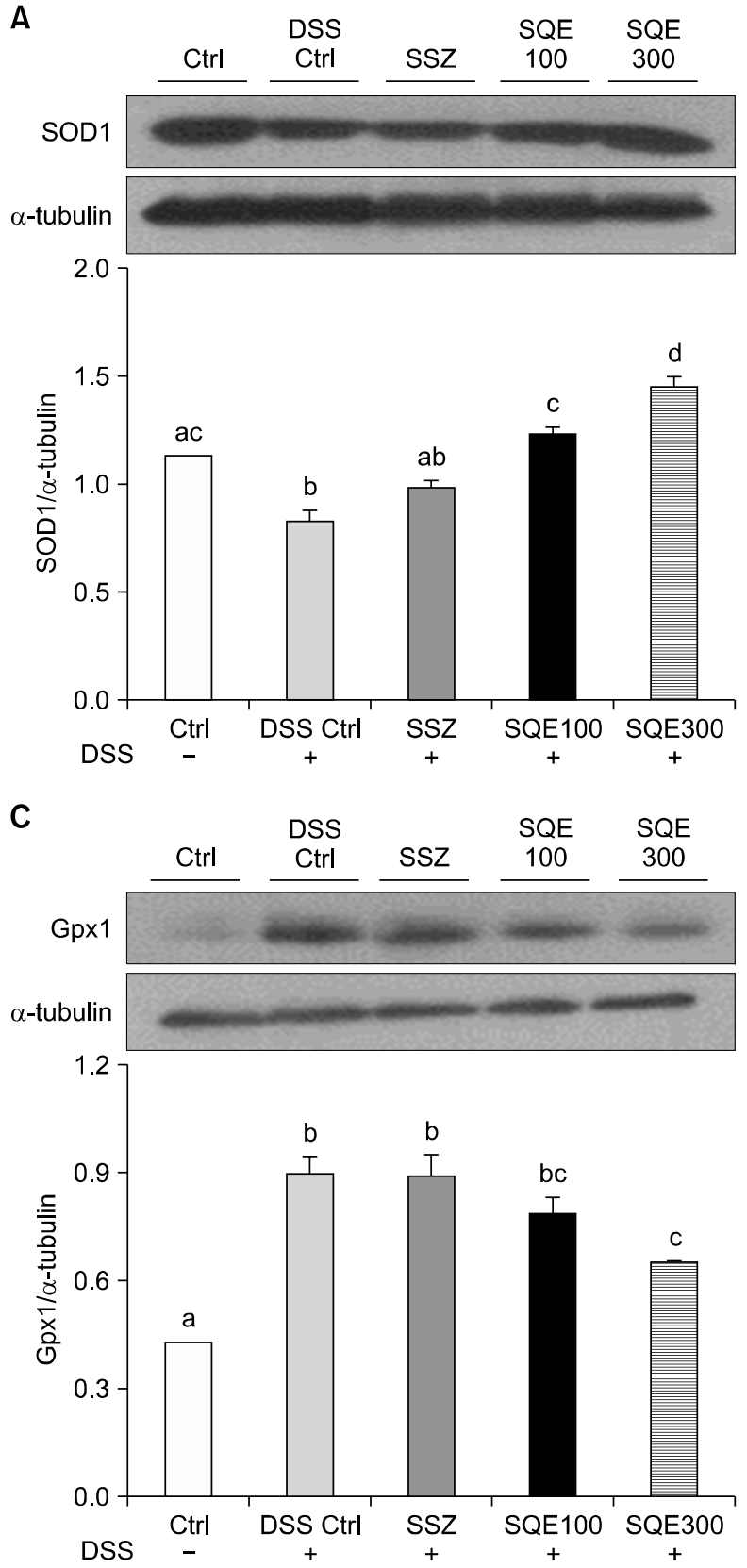

cells are associated with poor survival in colorectal cancer patients. In the present study, higher levels of 8-oxo-dG were detected in the DSS Ctrl group, and these results are consistent with those of recent studies where higher levels of DNA damage were associated with the production of ROS as part of the inflammation response in a DSS-induced colitis model. ${ }^{42,43}$ In contrast, SQE treatment resulted in the lower levels of 8-oxo-dG in the colon cells examined. Taken together, these results indicate that SQE protects cells from DNA damage resulting from oxidative stress.

ROS are produced by activated neutrophils and macrophage and can be assessed by measuring the levels of MDA in colon
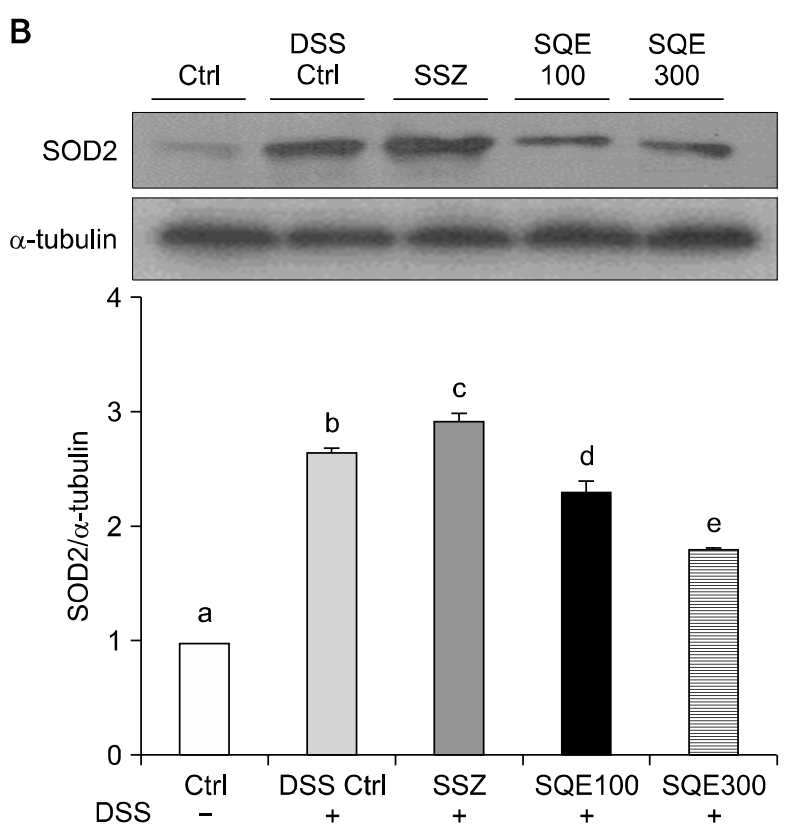

Figure 5. SQE administration regulates expression of SOD and Gpx1 in the colon of mice treated with DSS. Expressions of SOD1 (A), SOD2 (B), and Gpx1 (C) were analyzed in colon tissues by using western blot. Level of $\alpha$-tubulin was detected as a loading control. Band intensities were quantified by densitometry and data shown are the mean \pm SEM and were used were analyzed using one-way ANOVA and Tukey's post-hoc test $(P<0.05) ; \mathrm{n}=10$ mice per group. Different letters are used to indicate significant differences. $\mathrm{SQE}$, Sasa quelpaertensis extract; SOD, superoxide dismutase; Gpx1, glutathione peroxidase 1; Ctrl, control; DSS, dextran sulfate sodium; SSZ, sulfasalazine.

tissues. ${ }^{44} \mathrm{MDA}$ levels increase in the presence of ROS thereby lead to lipid peroxidation. ${ }^{38}$ Mice treated with DSS have been found to have elevated MDA level. ${ }^{45}$ Also, Rise et al. ${ }^{46}$ reported that levels of MDA were increased in the colon of an UC mouse model that was induced by acetic acid. In the present study, MDA levels were higher in the DSS Ctrl group, whereas the SQE group exhibited a significant decrease in MDA levels. These results indicate that administration of SQE may reduce MDA activity in plasma by protecting the lipid peroxidation process.

Activation of phagocytic leukocytes is induced by inflammation in the mucosa, and the production of ROS increases, including 
that of superoxide anions. ${ }^{3}$ Uncontrolled overproduction of ROS can disturb protective mechanisms and can lead to cellular oxidative damage. ${ }^{47} \mathrm{SOD}$ is an antioxidant enzyme that converts the highly reactive superoxide anion, $\mathrm{O}_{2}{ }^{-}$, to less reactive species such as $\mathrm{H}_{2} \mathrm{O}_{2}$ and $\mathrm{O}_{2}{ }^{10}$ During inflammation, high levels of ROS can disturb SOD antioxidant enzymes and decrease SOD enzyme activity, as observed in IBD patients with active disease. ${ }^{16}$ In the present study, SOD activity was significantly reduced in the plasma samples obtained from the DSS Ctrl group, whereas SOD activity was maintained following administration of SQE. It is possible that the latter observation may contribute to reduced tissue damage.

In mammalian cells, three isoforms of SOD have been identified: SOD1 (Cu-Zn SOD), SOD2 (Mn SOD), and SOD3 (ExtracellularSOD) ${ }^{48}$ In the present study, the effect of SQE on the expression of SOD1 and SOD2 was studied in a DSS-induced inflammation animal model. SOD1 (Cu-Zn SOD) is considered to have an important role in the first line of antioxidant defense. SOD1 is also located throughout the cytoplasm, yet is absent in mitochondria. ${ }^{47}$ Previous studies have shown that SOD1 protein expression and activity are decreased in inflamed mucosa from IBD patients. ${ }^{7,49}$ Moreover, high levels of ROS have been reported to disturb SOD1 antioxidant enzymes during inflammation. In the present study, lower levels of SOD1 expression were consistently detected in the DSS Ctrl group compared with the Ctrl group, whereas higher levels of SOD1 expression were detected in the administration of SQE group.

In contrast with SOD1, SOD2 (Mn-SOD) exclusively localized to mitochondria, and oxygen radicals in the respiratory chain are removed by SOD2 ${ }^{47}$ It has been suggested that SOD2 is a new type of tumor suppressor gene..$^{50}$ Unlike SOD1, SOD2 is easily induced and is up-regulated under inflammatory conditions following exposure to various oxidants. Thus, levels of SOD2 are increased under inflammation conditions involving ROS ${ }^{51.52}$ In the present study, expression levels of SOD2 were consistently up-regulated in the DSS group, whereas these levels were suppressed with administration of SQE. These results suggest that administration of SOE regulates expression of SOD antioxidant enzymes under inflammatory conditions.

Gpx and catalase normally remove $\mathrm{H}_{2} \mathrm{O}_{2}$ and share $\mathrm{H}_{2} \mathrm{O}_{2}$ as substrate, however both enzymes have different features. Gpx localizes to intestinal epithelial cells and acts as a primary defense against low concentrations of $\mathrm{H}_{2} \mathrm{O}_{2} .{ }^{53}$ Iantomasi et al. ${ }^{54}$ reported that the mucosa of IBD patients' exhibited higher levels of Gpx activity. In the present study, higher levels of Gpx expression were detected in colon tissues from the DSS Ctrl group compared with colon tissues from the Ctrl group, and the levels of Gpx were suppressed in tissues from the SQE group. Catalase efficiently converts $\mathrm{H}_{2} \mathrm{O}_{2}$ to water and $\mathrm{O}_{2}$ in peroxisomes and also protects cells from oxidative stress due to $\mathrm{H}_{2} \mathrm{O}_{2} \cdot{ }^{55}$ Experimental studies have shown that exposure to ROS can lead to a rapid inhibition of catalase, but not Gpx activity. ${ }^{5.57}$ In the present study, lower levels of catalase expression were detected in the DSS Ctrl group tissues compared to the Ctrl group, whereas the levels of catalase expression were higher in the tissues from the SQE group. Taken together, these results provide further evidence that SQE has the ability to restore balance among the antioxidant enzymes that are affected by an oxidative stress environment and negatively affect IBD progression.

Many studies have reported that natural polyphenols provide beneficial effects in colitis models. ${ }^{58.59}$ Accordingly, recent studies have reported that various Sasa leaves and their bioactive compounds mediate antioxidant effects against oxidative stress. ${ }^{60.63}$ For example, one of major components of SQE, $p$-coumaric acid, has been shown to mediate an antioxidant effect on low-density lipoprotein cholesterol oxidation. ${ }^{63}$ However, the antioxidant effects of individual bioactive compounds of SQE were not analyzed in the present study. Therefore, further studies are warranted to investigate the antioxidant properties of the individual bioactive compounds of SQE in IBD models.

In conclusion, the results of the present study indicate that SQE can mediate an inhibition of IBD progression and a reduction in DNA damage in a mouse model of DSS-induced colitis. Furthermore, SQE can regulate antioxidant enzymes that play key roles in the antioxidant defense system of a cell. Insight into the mechanistic details of these processes will improve our understanding of the antioxidant effect of SQE and will facilitate development of the natural therapeutic strategies for IBD patients.

\section{ACKNOWLEDGMENTS}

This work conduced with the support of the "Cooperative Research Program for Agriculture Science \& Technology Development (Project No, PJ009777)" Rural Development Administration, Republic of Korea and the Brain Korea 21 Plus (Project No. 22A20130012143).

\section{CONFLICTS OF INTEREST}

No potential conflicts of interest were disclosed. 


\section{REFERENCES}

1. Sands BE. Inflammatory bowel disease: past, present, and future. J Gastroenterol 2007:42:16-25.

2. Baumgart DC, Carding SR. Inflammatory bowel disease: cause and immunobiology. Lancet 2007;369:1627-40.

3. Zhu H, Li YR. Oxidative stress and redox signaling mechanisms of inflammatory bowel disease: updated experimental and clinical evidence. Exp Biol Med (Maywood) 2012;237:474-80.

4. Pani G, Galeotti T, Chiarugi P. Metastasis: cancer cell's escape from oxidative stress. Cancer Metastasis Rev 2010;29:351-78.

5. Debnath T, Kim da H, Lim BO. Natural products as a source of anti-inflammatory agents associated with inflammatory bowel disease. Molecules 2013;18:7253-70.

6. Kruidenier L, Kuiper I, Lamers CB, Verspaget HW. Intestinal oxidative damage in inflammatory bowel disease: semi-quantification, localization, and association with mucosal antioxidants. J Pathol 2003;201:28-36.

7. Lih-Brody L, Powell SR, Collier KP, Reddy GM, Cerchia R, Kahn E, et al. Increased oxidative stress and decreased antioxidant defenses in mucosa of inflammatory bowel disease. Dig Dis Sci 1996:41:2078-86.

8. Simmonds NJ, Allen RE, Stevens TR, Van Someren RN, Blake DR, Rampton DS. Chemiluminescence assay of mucosal reactive oxygen metabolites in inflammatory bowel disease. Gastroenterology 1992;103:186-96.

9. Kaspar JW, Niture SK, Jaiswal AK. Nrf2:INrf2 (Keap1) signaling in oxidative stress. Free Radic Biol Med 2009;47:1304-9.

10. Fridovich I. Superoxide radical and superoxide dismutases. Annu Rev Biochem 1995;64:97-112.

11. Pravda J. Radical induction theory of ulcerative colitis. World J Gastroenterol 2005;11:2371-84.

12. Kruidenier L, Kuiper I, van Duijn W, Marklund SL, van Hogezand RA, Lamers CB, et al. Differential mucosal expression of three superoxide dismutase isoforms in inflammatory bowel disease. J Pathol 2003;201:7-16.

13. Kruidenier L, Kuiper I, Van Duijn W, Mieremet-Ooms MA, van Hogezand RA, Lamers CB, et al. Imbalanced secondary mucosal antioxidant response in inflammatory bowel disease. J Pathol 2003;201:17-27.

14. Melgar S, Karlsson L, Rehnström E, Karlsson A, Utkovic H, Jansson L, et al. Validation of murine dextran sulfate sodium-induced colitis using four therapeutic agents for human inflammatory bowel disease. Int Immunopharmacol 2008;8:836-44.

15. Rose WA 2nd, Sakamoto K, Leifer CA. Multifunctional role of dextran sulfate sodium for in vivo modeling of intestinal diseases. BMC Immunol 2012;13:41.

16. Kruidenier L, Verspaget HW. Review article: oxidative stress as a pathogenic factor in inflammatory bowel disease: radicals or ridiculous? Aliment Pharmacol Ther 2002;16:1997-2015.

17. Cottone M, Renna S, Orlando A, Mocciaro F. Medical management of Crohn's disease. Expert Opin Pharmacother 2011;12: 2505-25.

18. Linares V, Alonso V, Domingo JL. Oxidative stress as a mechanism underlying sulfasalazine-induced toxicity. Expert Opin Drug Saf 2011;10:253-63.

19. Uko V, Thangada S, Radhakrishnan K. Liver disorders in inflammatory bowel disease. Gastroenterol Res Pract 2012;2012:
642923.

20. González-Gallego J, García-Mediavilla MV, Sánchez-Campos S, Tuñón MJ. Fruit polyphenols, immunity and inflammation. Br J Nutr 2010;104 Suppl 3:S15-27.

21. Romier B, Schneider YJ, Larondelle Y, During A. Dietary polyphenols can modulate the intestinal inflammatory response. Nutr Rev 2009;67:363-78.

22. Lenoir L, Rossary A, Joubert-Zakeyh J, Vergnaud-Gauduchon J, Farges MC, Fraisse D, et al. Lemon verbena infusion consumption attenuates oxidative stress in dextran sulfate sodium-induced colitis in the rat. Dig Dis Sci 2011:56:3534-45.

23. Boeing H, Bechthold A, Bub A, Ellinger S, Haller D, Kroke A, et al. Critical review: vegetables and fruit in the prevention of chronic diseases. Eur J Nutr 2012;51:637-63.

24. Okabe S, Takeuchi K, Takagi K, Shibata M. Stimulatory effect of the water extract of bamboo grass (Folin solution) on gastric acid secretion in pylorus-ligated rats. Jpn J Pharmacol 1975;25:608-9.

25. Kang SI, Shin HS, Kim HM, Hong YS, Yoon SA, Kang SW, et al. Anti-obesity properties of a Sasa quelpaertensis extract in high-fat diet-induced obese mice. Biosci Biotechnol Biochem 2012;76:755-61.

26. Kang SW, Kang SI, Shin HS, Yoon SA, Kim JH, Ko HC, et al. Sasa quelpaertensis Nakai extract and its constituent p-coumaric acid inhibit adipogenesis in 3T3-L1 cells through activation of the AMPK pathway. Food Chem Toxicol 2013;59:380-5.

27. Moon JY, Yang EJ, Kim SS, Kang JY, Kim GO, Lee NH, et al. Sasa quelpaertensis phenylpropanoid derivative suppresses lipopolysaccharide-induced nitric oxide synthase and cyclo-oxygenase-2 expressions in RAW 264.7 cells. Yakugaku Zasshi 2011;131: 961-7.

28. Kim S, Moustaid-Moussa N. Secretory, endocrine and autocrine/paracrine function of the adipocyte. J Nutr 2000;130: 3110S-5S.

29. Kim S, Urs S, Massiera F, Wortmann P, Joshi R, Heo YR, et al. Effects of high-fat diet, angiotensinogen (agt) gene inactivation, and targeted expression to adipose tissue on lipid metabolism and renal gene expression. Horm Metab Res 2002;34:721-5.

30. Kim KM, Kim YS, Lim JY, Min SJ, Shin JH, Ko HC, et al. Sasa quelpaertensis leaf extract suppresses dextran sulfate sodium-induced colitis in mice by inhibiting the proinflammatory mediators and mitogen-activated protein kinase phosphorylation. Nutr Res 2014:34:894-905.

31. Dieleman LA, Palmen MJ, Akol H, Bloemena E, Peña AS, Meuwissen SG, et al. Chronic experimental colitis induced by dextran sulphate sodium (DSS) is characterized by Th1 and Th2 cytokines. Clin Exp Immunol 1998;114:385-91.

32. Li Z, Chalazonitis A, Huang YY, Mann JJ, Margolis KG, Yang QM, et al. Essential roles of enteric neuronal serotonin in gastrointestinal motility and the development/survival of enteric dopaminergic neurons. J Neurosci 2011;31:8998-9009.

33. Sheridan J, Wang LM, Tosetto M, Sheahan K, Hyland J, Fennelly $D$, et al. Nuclear oxidative damage correlates with poor survival in colorectal cancer. Br J Cancer 2009;100:381-8.

34. Goyal N, Rana A, Ahlawat A, Bijjem KR, Kumar P. Animal models of inflammatory bowel disease: a review. Inflammopharmacology 2014:22:219-33.

35. Pavlick KP, Laroux FS, Fuseler J, Wolf RE, Gray L, Hoffman J, et al. Role of reactive metabolites of oxygen and nitrogen in inflammatory bowel disease. Free Radic Biol Med 2002;33:311-22. 
36. Rainis T, Maor I, Lanir A, Shnizer S, Lavy A. Enhanced oxidative stress and leucocyte activation in neoplastic tissues of the colon. Dig Dis Sci 2007;52:526-30.

37. Oz HS, Chen TS, McClain CJ, de Villiers WJ. Antioxidants as novel therapy in a murine model of colitis. J Nutr Biochem 2005;16: 297-304.

38. Yao J, Wang JY, Liu L, Li YX, Xun AY, Zeng WS, et al. Anti-oxidant effects of resveratrol on mice with DSS-induced ulcerative colitis. Arch Med Res 2010:41:288-94.

39. Oz HS, Chen T, de Villiers WJ. Green tea polyphenols and sulfasalazine have parallel anti-inflammatory properties in colitis models. Front Immunol 2013:4:132.

40. Osada T, Ohkusa T, Okayasu I, Yoshida T, Hirai S, Beppu K, et al. Correlations among total colonoscopic findings, clinical symptoms, and laboratory markers in ulcerative colitis. J Gastroenterol Hepatol 2008;23 Suppl 2:S262-7.

41. Dincer Y, Erzin Y, Himmetoglu S, Gunes KN, Bal K, Akcay T. Oxidative DNA damage and antioxidant activity in patients with inflammatory bowel disease. Dig Dis Sci 2007:52:1636-41.

42. Barollo M, Medici V, D'Incà R, Banerjee A, Ingravallo G, Scarpa M, et al. Antioxidative potential of a combined therapy of anti TNF $\alpha$ and $\mathrm{Zn}$ acetate in experimental colitis. World J Gastroenterol 2011:17:4099-103.

43. Trivedi PP, Jena GB. Melatonin reduces ulcerative colitisassociated local and systemic damage in mice: investigation on possible mechanisms. Dig Dis Sci 2013;58:3460-74.

44. Kim YJ, Kim EH, Hahm KB. Oxidative stress in inflammationbased gastrointestinal tract diseases: challenges and opportunities. J Gastroenterol Hepatol 2012;27:1004-10.

45. Ajayi BO, Adedara IA, Farombi EO. Pharmacological activity of 6-gingerol in dextran sulphate sodium-induced ulcerative colitis in BALB/c mice. Phytother Res 2015:29:566-72.

46. Rise CL, Prabhu VV, Guruvayoorappan C. Effect of marine mangrove Avicennia marina (Forssk.) Vierh against acetic acid-induced ulcerative colitis in experimental mice. J Environ Pathol Toxicol Oncol 2012;31:179-92.

47. Matés JM, Pérez-Gómez C, Núñez de Castro I. Antioxidant enzymes and human diseases. Clin Biochem 1999;32:595-603.

48. Sandström J, Nilsson P, Karlsson K, Marklund SL. 10-fold increase in human plasma extracellular superoxide dismutase content caused by a mutation in heparin-binding domain. J Biol Chem 1994:269:19163-6.

49. Dagli U, Balk M, Yücel D, Ulker A, Over H, Saydam G, et al. The role of reactive oxygen metabolites in ulcerative colitis. Inflamm Bowel Dis 1997:3:260-4

50. Bravard A, Sabatier L, Hoffschir F, Ricoul M, Luccioni C,
Dutrillaux B. SOD2: a new type of tumor-suppressor gene? Int J Cancer 1992;51:476-80.

51. Röhrdanz E, Kahl R. Alterations of antioxidant enzyme expression in response to hydrogen peroxide. Free Radic Biol Med 1998:24:27-38.

52. Jackson RM, Parish G, Helton ES. Peroxynitrite modulates MnSOD gene expression in lung epithelial cells. Free Radic Biol Med 1998;25:463-72.

53. Tham DM, Whitin JC, Kim KK, Zhu SX, Cohen HJ. Expression of extracellular glutathione peroxidase in human and mouse gastrointestinal tract. Am J Physiol 1998;275:G1463-71.

54. Iantomasi T, Marraccini P, Favilli F, Vincenzini MT, Ferretti $P$, Tonelli F. Glutathione metabolism in Crohn's disease. Biochem Med Metab Biol 1994:53:87-91.

55. Singh AK, Dhaunsi GS, Gupta MP, Orak JK, Asayama K, Singh I. Demonstration of glutathione peroxidase in rat liver peroxisomes and its intraorganellar distribution. Arch Biochem Biophys 1994:315:331-8.

56. Kono Y, Fridovich I. Superoxide radical inhibits catalase. J Biol Chem 1982;257:5751-4.

57. Pigeolet E, Corbisier P, Houbion A, Lambert D, Michiels C, Raes $\mathrm{M}$, et al. Glutathione peroxidase, superoxide dismutase, and catalase inactivation by peroxides and oxygen derived free radicals. Mech Ageing Dev 1990;51:283-97.

58. Rosillo MA, Sanchez-Hidalgo M, Cárdeno A, de la Lastra CA. Protective effect of ellagic acid, a natural polyphenolic compound, in a murine model of Crohn's disease. Biochem Pharmacol 2011;82:737-45.

59. Sánchez-Fidalgo S, Cárdeno A, Sánchez-Hidalgo M, Aparicio-Soto M, de la Lastra CA. Dietary extra virgin olive oil polyphenols supplementation modulates DSS-induced chronic colitis in mice. J Nutr Biochem 2013:24:1401-13.

60. Lu B, Wu X, Shi J, Dong Y, Zhang Y. Toxicology and safety of antioxidant of bamboo leaves. Part 2: developmental toxicity test in rats with antioxidant of bamboo leaves. Food Chem Toxicol 2006:44:1739-43.

61. Park HS, Lim JH, Kim HJ, Choi HJ, Lee IS. Antioxidant flavone glycosides from the leaves of Sasa borealis. Arch Pharm Res 2007:30:161-6.

62. Gong J, Xia D, Huang J, Ge Q, Mao J, Liu S, et al. Functional components of bamboo shavings and bamboo leaf extracts and their antioxidant activities in vitro. J Med Food 2015;18:453-9.

63. Zang LY, Cosma G, Gardner H, Shi X, Castranova V, Vallyathan V. Effect of antioxidant protection by p-coumaric acid on low-density lipoprotein cholesterol oxidation. Am J Physiol Cell Physiol 2000;279:C954-60. 